\title{
Preparation and Characterization of Biocompatible Polymer Particles as Potential Nanocarriers for Inhalation Therapy
}

\author{
Katarzyna Jabłczyńska, Magdalena Janczewska, \\ Aleksandra Kulikowska, and Tomasz R. Sosnowski \\ Faculty of Chemical and Process Engineering, Warsaw University of Technology, Waryńskiego 1, 00-645 Warsaw, Poland
}

Correspondence should be addressed to Tomasz R. Sosnowski; t.sosnowski@ichip.pw.edu.pl

Received 1 January 2015; Accepted 6 February 2015

Academic Editor: Jan-Chan Huang

Copyright (C) 2015 Katarzyna Jabłczyńska et al. This is an open access article distributed under the Creative Commons Attribution License, which permits unrestricted use, distribution, and reproduction in any medium, provided the original work is properly cited.

\begin{abstract}
Aim. Investigation of the possibility of manufacturing biocompatible polymer particles which have the required properties for pulmonary delivery via inhalation and simultaneously act as vehicles of nanotherapeutics. Methods. Nanostructures were obtained from biocompatible polysaccharides by successive oxidation and reactive coiling in the aqueous phase. The resultant nanosuspensions of PAD (polyaldehyde dextran) and DACMC (dialdehyde carbomethylcellulose) were used as precursors in spray drying production of powders at variable process conditions. The resultant dry microparticles were characterized by SEM observations, and their properties related to delivery by inhalation were determined by laser diffraction spectrometry following the dispersion in the commercial inhaler. Finally, the possibility of the reconstitution of nanosuspensions by powders rehydration was evaluated. Results. Synthesized nanoparticles had size of 120-170 nm. Microparticles after drying had size of $0.5-5 \mu \mathrm{m}$ and different surface morphology. Aerosolized particles obtained from powder dispersion in the inhaler had the volumetric median diameter of $\sim 2$ and $\sim 1 \mu \mathrm{m}$ for PAD and DACMC, respectively. Hydration of powders led to restoring the nanosuspensions with the average particle size similar to the precursor. Conclusions. PAD and DACMC can be used to obtain nanostructures which, after processing, take a form suitable for effective delivery to the lungs via inhalation.
\end{abstract}

\section{Introduction}

Biocompatible and biodegradable polymeric particles are widely used as drug carriers [1-4]. Encapsulation of drug in polymer particles allows for prolonged release of medicines in the target area which means that drugs become available directly in the desired site (so they do not cause side effects) and their activity is extended in time. Typically, drug release is achieved due to polymer swelling or erosion [5]. In addition, the release rate can be "tuned" using the responsive systems; that is, it can be triggered by $\mathrm{pH}$, temperature, radiation, magnetic field, and so forth $[6,7]$. In such cases polymerbased carriers become the specialized and effective tool of medical treatment. Simultaneously, the controlled release allows minimizing local toxic effects of potent drugs [4].

Nanoparticles gained a lot of interest as potential vehicles of therapeutics because of several unique and beneficial features. Owing to the small area for opsonin binding, they are capable of avoiding the phagocytosis so the drug bioavailability is increased as compared to larger carriers [5]. Simultaneously, the size of nanoparticles makes them suitable to penetrate to the tumors via more leaky walls of the blood vessels (so called, EPR effect) and for this reason they are preferable carriers of anticancer therapeutics $[8,9]$.

The common route of delivery of drugs formed as micro- and nanoparticles is intravenous injection; however pulmonary drug delivery by inhalation becomes prospective [10-12]. It is beneficial particularly in the treatment of disorders localized within the respiratory system where diseased areas can be accessed from the outer side (i.e., via the air). Unfortunately, nanoparticles cannot be efficiently delivered by inhalation due to their poor aerosolization properties and relatively ineffective deposition in the bronchial tree and the alveoli. According to the existing knowledge based simultaneously on in vivo measurements and model calculations, the most suitable aerodynamic size of aerosol particles which 
assures their good penetration to the lower airways of the respiratory system is limited to $1-5 \mu \mathrm{m}$ [12-14]. Thus, delivery of nanodrugs or nanocarriers into the lungs requires an application of additional vehicles of nanostructures in form of microsized objects which can be inhaled and effectively deposited in the respiratory system $[10,15,16]$.

Typical polymers considered for use in pulmonary drug delivery are natural polysaccharides such as carrageenans, chitosan, gelatin, and hyaluronic acid, but also certain biocompatible synthetic compounds such as poly(lactic) acid, PLA; oligo(lactic) acid polymers, poly(vinyl) alcohol, PVA; and also some copolymers (e.g., poly(lactic-co-glycolide acid): PLGA) [5]. Other biocompatible polymers may be also proposed as the field is still open for innovation [17]. Such compounds can be used as carriers of drugs which are incorporated in the dissolving matrix. One of the key problems in the application of respirable drug carriers is their preparation as a formulation suitable for use with inhalers. Among several available methods of production of stable dry particles [18], spray drying (SD) became popular as a single-step and affordable process [19]. Other advantages of this technique are easy scaling up, good control over product quality (mainly: particle size and morphology), and the possibility of production of multicomponent particles [20-22].

In this paper we focus on the preparation of inhalable microparticles which can be used as vehicles for nanosized polymeric particles for delivery to the respiratory system. To achieve that aim we propose the multistep research approach which consists of the follwoing:

(1) preparation of nanoparticles by oxidized dextran and carboxymethyl-cellulose (both compounds are known to be biocompatible $[23,24]$, and this property should be retained after oxidation as they are always partially oxidized in the native state),

(2) preparation of microparticles by SD starting from the nanosuspensions obtained in step (1),

(3) characterization of the microparticles (powders) in respect of their aerosolization properties to confirm suitability for pulmonary drug delivery,

(4) testing the possibility of reconstitution of nanostructures after powder hydration.

\section{Materials and Methods}

2.1. Nanoparticles Preparation and Size Determination. The following chemicals have been used in the synthesis of nanoparticles: dextran $(\mathrm{MW}=70 \mathrm{kDa}$, donated by Institute of Animal Physiology and Nutrition, Polish Academy of Sciences, Jablonna, Poland), Aqualon sodium carboxymethylcellulose (MW $=90 \mathrm{kDA}$, Hercules Inc., USA), dodecylamine hydrochloride, benzylamine, sodium periodate (Sigma Aldrich, Poland), and alanine (POCh, Poland).

Each of two polysaccharides was oxidized in aqueous phase with sodium periodate according to procedure described by $\mathrm{Mu}$ et al. [25]. Degree of oxidation after the completion of the reaction was determined by hydroxylamine hydrochloride titration method [26], and it was 5\% in the case of
TABLE 1: Processing conditions of spray drying: different flow rates and temperatures of the drying air.

\begin{tabular}{lccc}
\hline & $\mathrm{Q}=20 \mathrm{~m}^{3} / \mathrm{hr}$ & \\
Designation & Exp. 1 & Exp. 2 & Exp. 3 \\
\hline$T_{\text {in }}\left[{ }^{\circ} \mathrm{C}\right]$ & 100 & 150 & 200 \\
$T_{\text {out }}\left[{ }^{\circ} \mathrm{C}\right]$ & $\sim 45$ & $\sim 57$ & $\sim 68$ \\
\hline \multicolumn{4}{c}{$\mathrm{Q}=35 \mathrm{~m}^{3} / \mathrm{hr}$} \\
Designation & Exp. 4 & Exp. 5 & Exp. 6 \\
\hline$T_{\text {in }}\left[{ }^{\circ} \mathrm{C}\right]$ & 100 & 150 & 200 \\
$T_{\text {out }}\left[{ }^{\circ} \mathrm{C}\right]$ & $\sim 55$ & $\sim 85$ & $\sim 100$ \\
\hline
\end{tabular}

polyaldehyde dextran (PAD) and $15 \%$ in case of dialdehyde carboxymethylcellulose (DACMC). Folding of polymer chains was achieved by linking hydrophobic amines to the aldehyde groups: dodecylamine hydrochloride in the case of PAD and benzylamine in the case of DACMC. Other aldehyde groups were substituted by alanine. These reactions allowed obtaining nanoparticles which were then purified by $30 \mathrm{~min}$ dialysis done in tubular membranes made of regenerated cellulose (MWCO 14 000, Carl Roth GmbH + Co. KG, Germany) against water purified with the reverse osmosis. The outer liquid was agitated with the magnetic stirrer $(150 \mathrm{rpm})$. The size of obtained nanostructures was determined at room temperature by nanoparticle tracking analysis (NTA) using Nanosight LM10-HS device (Malvern Instruments, UK). Each nanosuspension (approximate concentration: $20 \mathrm{mg} / \mathrm{mL}$ ) was diluted 100 -fold with water before the measurements. Analyses were triplicated and the gathered data were averaged.

2.2. Powder Production and Characterization. Dry powder was produced with the standard spray drying method (SD) using Mini Spray Dryer B-290 (Büchi, Switzerland) operated in the open mode. Some details of the technique and the apparatus are given elsewhere [22]. The aqueous nanosuspensions (approximate concentration: PAD: 6\% and DACMC: $2 \%)$ were pumped to the pneumatic nozzle with the volumetric rate of $3 \mathrm{~mL} / \mathrm{min}$ and dispersed by compressed air (flow rate: $14 \mathrm{dm}^{3} / \mathrm{min}$ ). The powders formed due to evaporation of water from droplets on contact with hot air (inlet temperature, $T_{\text {in }}=100,150$, or $200^{\circ} \mathrm{C}$ ) were separated in the high performance glass cyclone. Two process parameters were adjusted: the flow rate $Q$ and the inlet temperature of the drying air $T_{\text {in }}$. These parameters were selected as decisive based on our previous studies related to use of spray drying for preparing of inhalable powders [20, 22]. Experimental settings of the drying are listed in Table 1. Collected powders were inspected visually using scanning electron microscope (SEM, model TM1000, Hitachi, Japan) after coating with $20 \mathrm{~nm}$ layer of gold (K550x Sputter Coater, Quorum Technologies, UK).

2.3. Aerosol Properties of Powders. Powder aerosolization was tested using the commercially available capsule inhaler (Cyclohaler, Adamed, Poland). Before each experiment capsules were loaded with approximately $10 \mathrm{mg}$ powder aliquots. Volumetric particle size distribution was determined with 


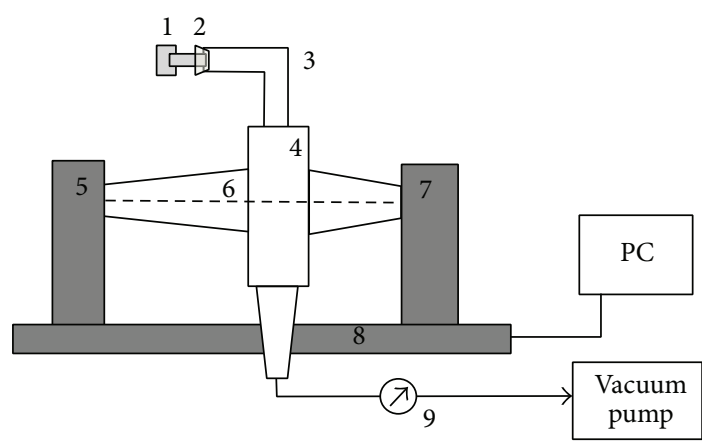

FIGURE 1: The experimental set-up for determination of particle size distribution of aerosolized powder: 1: inhaler, 2: adapter, 3: USP inlet, 4: inhalation chamber, 5: laser emitter of Spraytec spectrometer, 6: laser beam, 7: laser detector of Spraytec spectrometer, 8: optical bench, and 9: valve and air flowmeter.

the laser diffraction aerosol spectrometer (Spraytec, Malvern Instruments, UK) additionally equipped with the dedicated inhalation chamber, Figure 1. Aerosol was created by the airflow drawn through the inhaler at the rate of $100 \mathrm{dm}^{3} / \mathrm{min}$ which is the typical value obtained during medical inhalations with this type of inhaling device. The aerosol cloud was introduced into the inhalation chamber via the standard inlet tube (so called: USP, i.e., United States Pharmacopeia, inlet [27]). The cloud was automatically sensed by the optical system allowing determining the particle size distribution (measuring period: $0.5 \mathrm{~s}$, sampling rate: $1 \mathrm{kHz}$ ). Based on the complete, time-averaged size distributions, two essential factors were evaluated and compared: the volumetric median diameter $(\mathrm{VMD}=\mathrm{D} v 50)$ and the percentage of particles smaller than $4.7 \mu \mathrm{m}$ (so called, fine particle fraction: FPF). Both parameters are the essential quality indicators of aerosol produced by the inhalation drug delivery systems [12].

It should be noted here that laser diffraction allows to find the distribution based on particles volume which is, in general, not equivalent to the one based on the aerodynamic size (e.g., measured by cascade impactors [27]). By definition, aerodynamic particle diameter equals

$$
d_{\mathrm{ae}}=d_{p} \sqrt{\frac{\rho_{p}}{\xi \rho_{w}}},
$$

where $d_{p}$ is the equivalent geometric diameter of the particle, $\rho_{p}$ density of particle material, $\rho_{w}$ density of water, and $\xi$ the dynamic shape factor [16]. Divergence between both diameters becomes important only for highly porous particles (like those studied by Tsapis et al. [15]) or particles with very irregular shape (needles, fibers, fractal-like aggregates, and plates: $\xi \gg 1)$. For particles with virtually spherical shape $(\xi=$ 1) and density comparable to water, it can be claimed that both diameters are practically equivalent. This assumption remains also valid for particles studied in this work.

2.4. Nanoparticle Reconstitution after Powder Rehydration. In order to check if nanocolloids can be restored after rehydration of dry particles, the powders ( $20 \mathrm{mg}$ aliquots) were mixed with water $(1 \mathrm{~mL})$ and gently agitated by magnetic ministirrer for 30 minutes at room temperature (agitation speed: $300 \mathrm{~min}^{-1}$ ). Determination of particle size distribution in the suspension was done by the same methodology as for the initial assessment, that is, by NTA.

\section{Results and Discussion}

Total concentration of PAD and DACMC synthesized nanoparticles in the samples used in NTA analyses was in the range of $10^{8}$ particles $/ \mathrm{mL}$. The examples of size distributions obtained by NTA measurements for both types of nanoparticles are presented in Figure 2. The nanoparticles were polydisperse with the average particle size: $168.5 \pm 16.6 \mathrm{~nm}$ and the count mode: $107 \pm 1.9 \mathrm{~nm}$ in case of PAD and the average size: $118.0 \pm 0.7 \mathrm{~nm}$ and the count mode: $96.0 \pm 0.5 \mathrm{~nm}$ in case of DACMC. For PAD particles it is seen that the distribution is characterized by the main maximum corresponding to the modal value and the additional local peak which probably shows aggregates. To better describe the size distribution we introduce the span, which can be determined according to the formula:

$$
S=\frac{D_{90}-D_{10}}{D_{50}},
$$

where $D_{i}$ denotes the $i$ th percentile of the cumulative distribution. Span values provide data regarding the width (polydispersity) of the particle size distribution. The calculated values of $S$ were $1.16 \pm 0.04$ and $1.06 \pm 0.02$ for PAD and DCMC nanoparticles, respectively (these data are shown in Figure 8(b) - “sample 0").

SEM pictures of powders obtained by drying of nanosuspensions of polymeric nanoparticles are presented in Figures 3 and 4 . These pictures are presented to illustrate the average geometrical size and morphology of dried material (no statistical analysis of particle sizes was done based on SEM measurements). In case of PAD (Figure 3), powder particles are typically characterized by wrinkled surface and the degree of corrugation increases when the drying becomes faster. At the low flow rate of the drying air with $T_{\text {in }}=100^{\circ} \mathrm{C}-150^{\circ} \mathrm{C}$ $\left(Q=20 \mathrm{~m}^{3} / \mathrm{hr}\right.$ ), some spherical and smooth particles can be found as indicated by arrows in Figure 3. Such particles are formed when drying process is slow enough to allow the uniform evaporation of water from droplets. Higher drying temperatures and increased airflow rates are responsible for the formation of particles with a corrugated surface.

At very high drying rates (Exps 3, 5, and 6), powder particles are highly deformed and crumpled. This effect is due to the surface collapse when the outer solid crust (shell) is formed over the liquid content of the inner core of the droplet as suggested by other authors [28]. In such case, water evaporates through the crust leaving empty spaces behind so the crust becomes wrinkled as it collapses to fill these spaces, Figure 5(a).

Based on the presented SEM pictures it can be found that the range of particle size is similar independently of the drying rate. Evidently, particles are not of equal size due to the polydispersity of the sprayed droplets. All particles are in the 


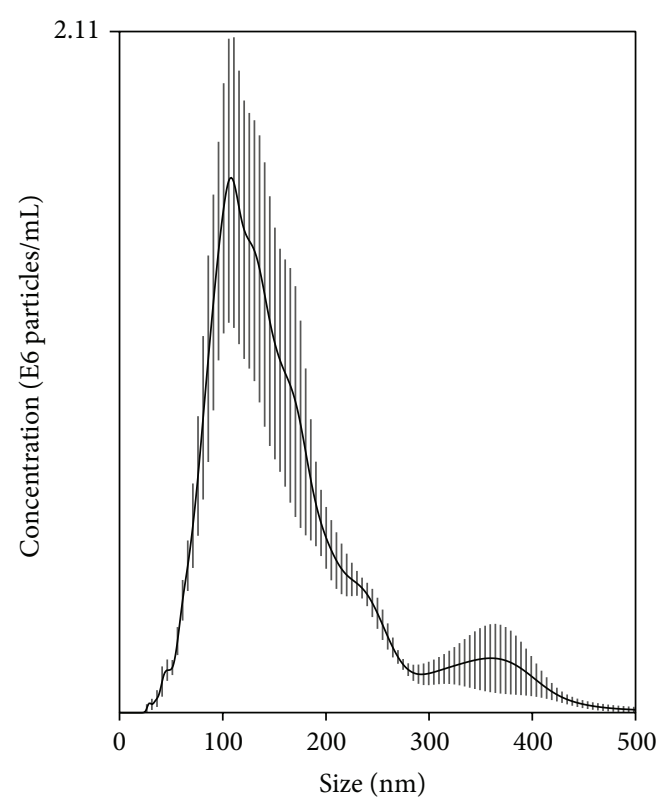

(a)

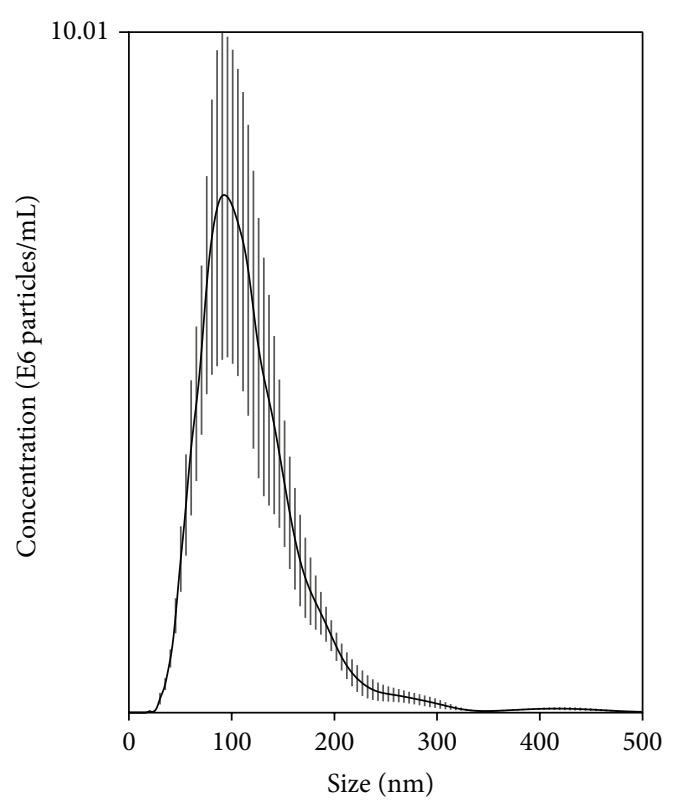

(b)

Figure 2: Examples of size distribution of PAD (a) and DACMC (b) nanoparticles.

PAD, Exp $1\left(100^{\circ} \mathrm{C}\right)$

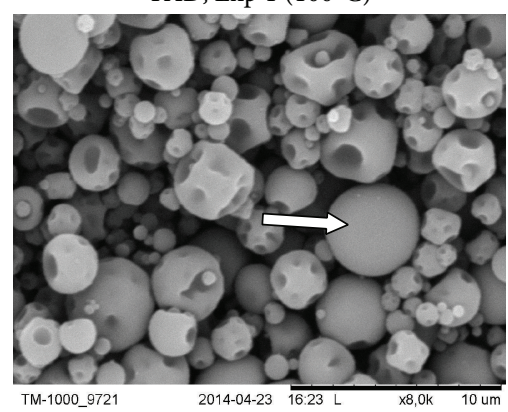

TM-1000_9721

2014-04-23 16.23 L $\quad \times 8,0 \mathrm{k} \quad 10 \mathrm{um}$
$Q=20 \mathrm{~m}^{3} / \mathrm{hr}$

PAD, $\operatorname{Exp} 2\left(150^{\circ} \mathrm{C}\right)$

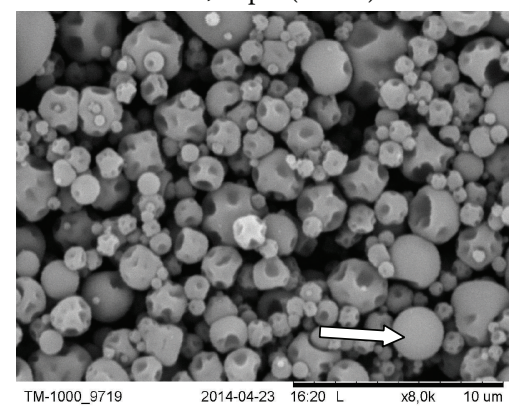

$Q=35 \mathrm{~m}^{3} / \mathrm{hr}$

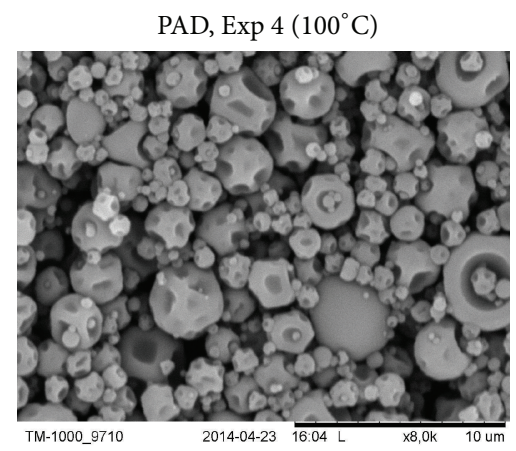

PAD, $\operatorname{Exp} 5\left(150^{\circ} \mathrm{C}\right)$

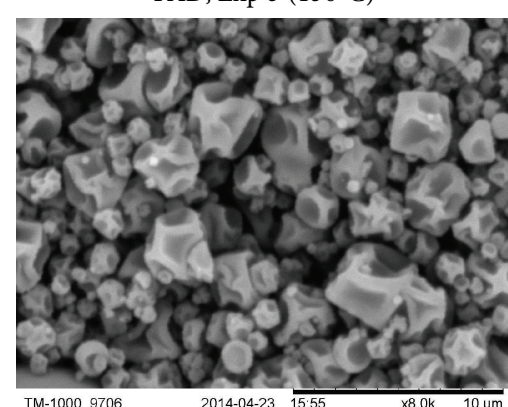

$\mathrm{PAD}, \operatorname{Exp} 3\left(200^{\circ} \mathrm{C}\right)$

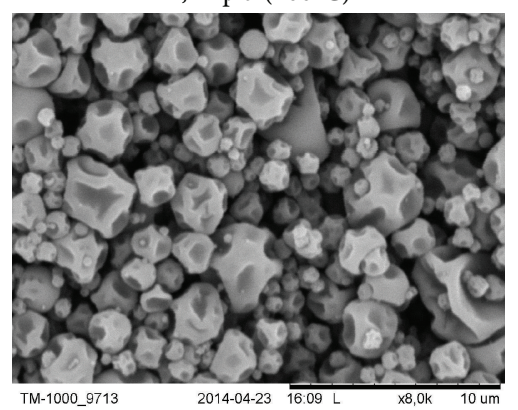

PAD, $\operatorname{Exp} 6\left(200^{\circ} \mathrm{C}\right)$

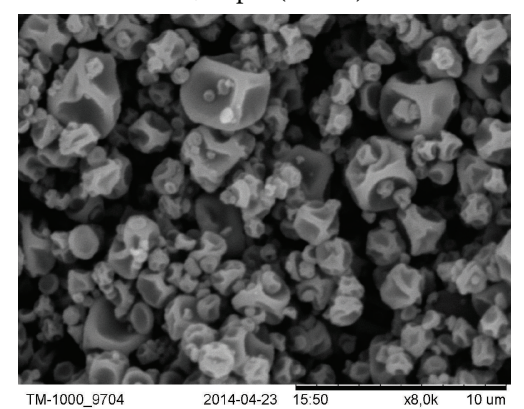

FIGURE 3: SEM pictures of powders obtained by spray drying of PAD nanosuspensions. Arrows indicate spherical particles with a smooth surface. 
$Q=20 \mathrm{~m}^{3} / \mathrm{hr}$

DACMC, $\operatorname{Exp} 1\left(100^{\circ} \mathrm{C}\right)$
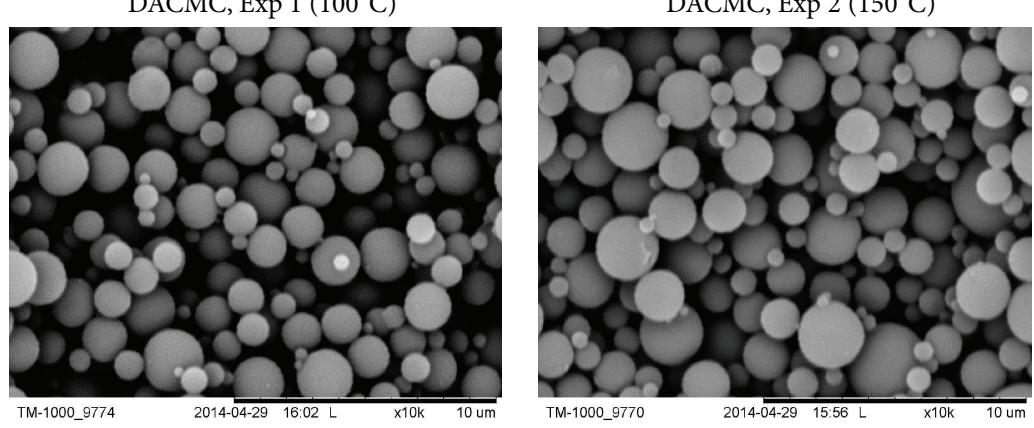

$Q=35 \mathrm{~m}^{3} / \mathrm{hr}$

DACMC, $\operatorname{Exp} 4\left(100^{\circ} \mathrm{C}\right)$

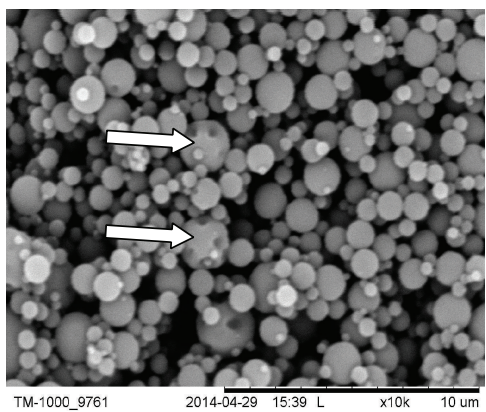

DACMC, $\operatorname{Exp} 5\left(150^{\circ} \mathrm{C}\right)$

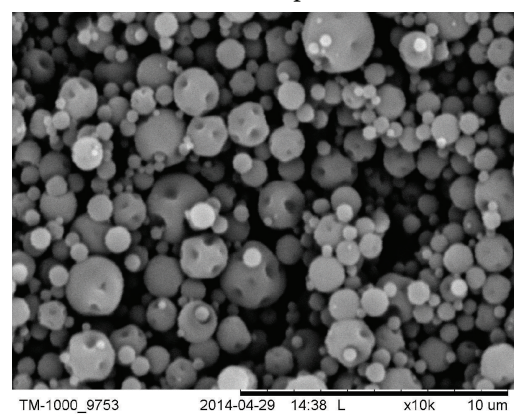

DACMC, $\operatorname{Exp} 3\left(200^{\circ} \mathrm{C}\right)$

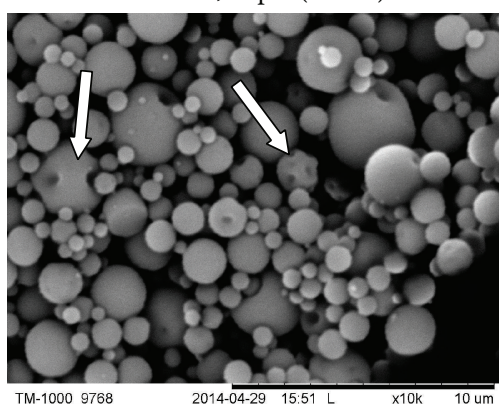

DACMC, $\operatorname{Exp} 6\left(200^{\circ} \mathrm{C}\right)$

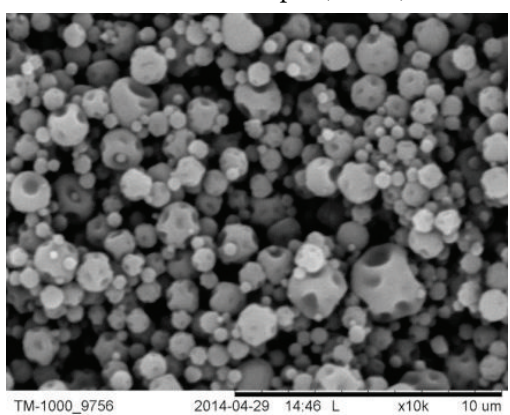

FIGURE 4: SEM pictures of powders obtained by spray drying of DACMC nanosuspensions. Arrows indicate deformed particles with a wrinkled surface.

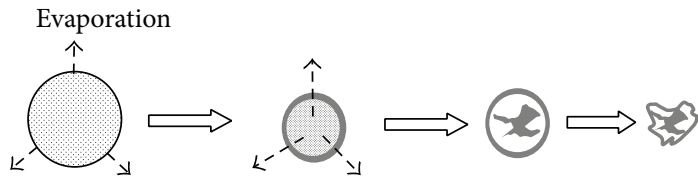

(a)

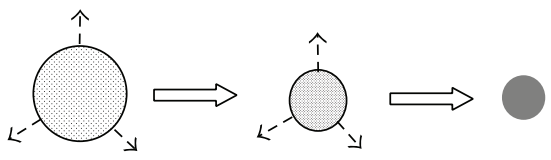

(b)

FIgURE 5: Hypothetical steps during formation of particles during drying: (a) at high drying rates formation of crust and evaporation of water from the inner liquid core leads to a deformed particle; (b) for low drying rate and/or lower concentration of the nanosuspension, droplets evaporate uniformly and the spherical particles are formed.

range of $0.5-5 \mu \mathrm{m}$, which is appropriate for powders designed for pulmonary delivery via inhalation.

In case of DACMC powders shown in Figure 4 the trend of changes in surface morphology is less apparent than the one observed for PAD particles.

At the lower flow rate of drying air $\left(Q=20 \mathrm{~m}^{3} / \mathrm{hr}\right)$ particles are formed as spherical and smooth. Some deformations of the surface are observed only at the highest drying temperature $\left(200^{\circ} \mathrm{C}\right)$ as indicated by arrow in this figure. When the flow rate of drying air increases, irregularities on particle surface become visible already at $T_{\text {in }}=100^{\circ} \mathrm{C}$. Particle corrugation is predominant at the highest drying rate but it is still much less remarkable when compared to PAD powders. It is expected that due to a lower concentration of the nanoparticles in the droplet, the mechanisms of drying is similar to the one shown in Figure 5(b), where the outer shell is not formed. It can be seen that DACMC particles are polydisperse and their size is typically below $3 \mu \mathrm{m}$. Higher flow rates of the drying air allows to obtain smaller particles.

Size distribution of particles dispersed in the air are compared in Figures 6 and 7, with a focus on two parameters: volumetric median diameter $(\mathrm{VMD}=\mathrm{Dv} 50)$ and the fraction of particles smaller than $4.7 \mu \mathrm{m}$ (fine particle fraction: FPF). As mentioned earlier, both the median diameter and the FPF are the most important parameters for the evaluation of aerosol suitability for drug delivery via the inhalative route [12].

The comparison shows that DACMC particles are at average almost twice smaller than PAD particles (VMD 1.1$1.5 \mu \mathrm{m}$ and $2-2.6 \mu \mathrm{m}$, resp.) and the average size (VMD) of aerosol particles noticeably depends on drying conditions. Smaller size of DACMC particles results from the lower concentration of the precursor nanosuspension than in the case of PAD. In spite of the discussed differences in size, both types of powders may be considered as very good candidates for carriers of inhaled drugs. Fine particle fraction (FPF) is 

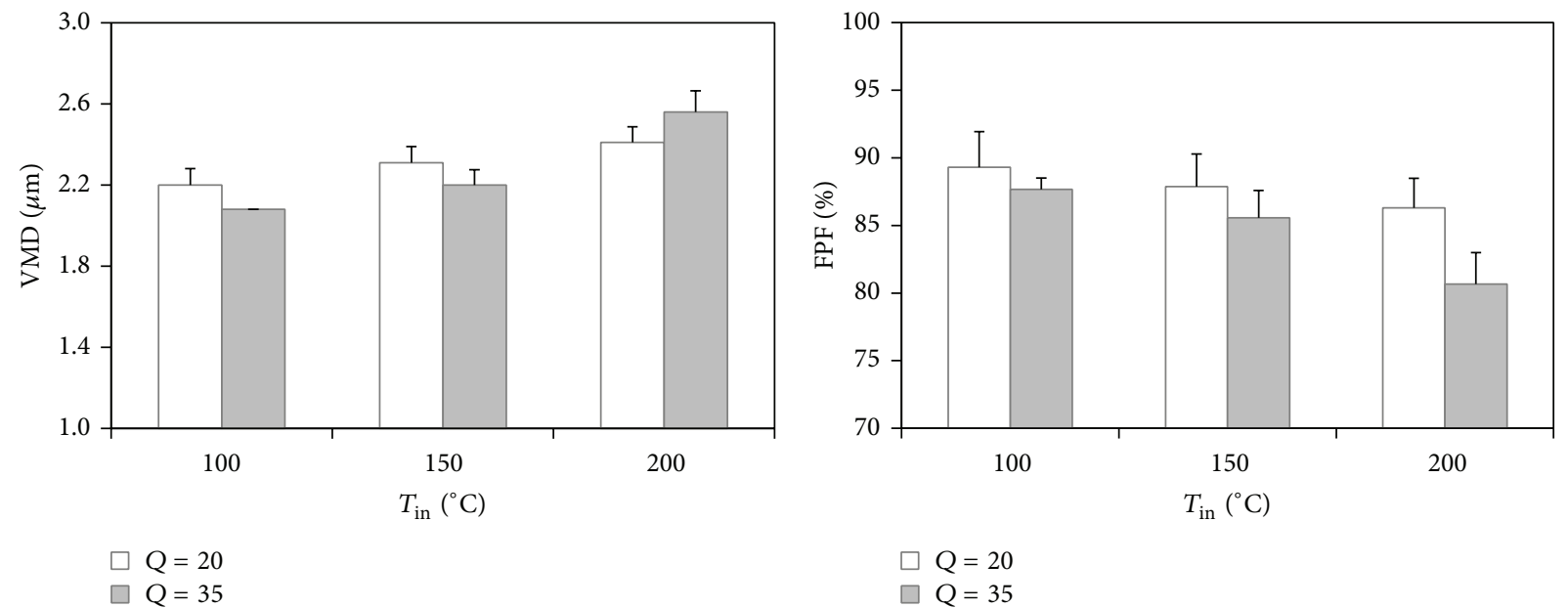

FIGURE 6: Volumetric median diameter (VMD) and fraction of fine particles (FPF) for PAD microparticles as a function of the temperature and the flow rate of the drying air $\left(\mathrm{m}^{3} / \mathrm{hr}\right)$. Error bars denote the standard deviation.
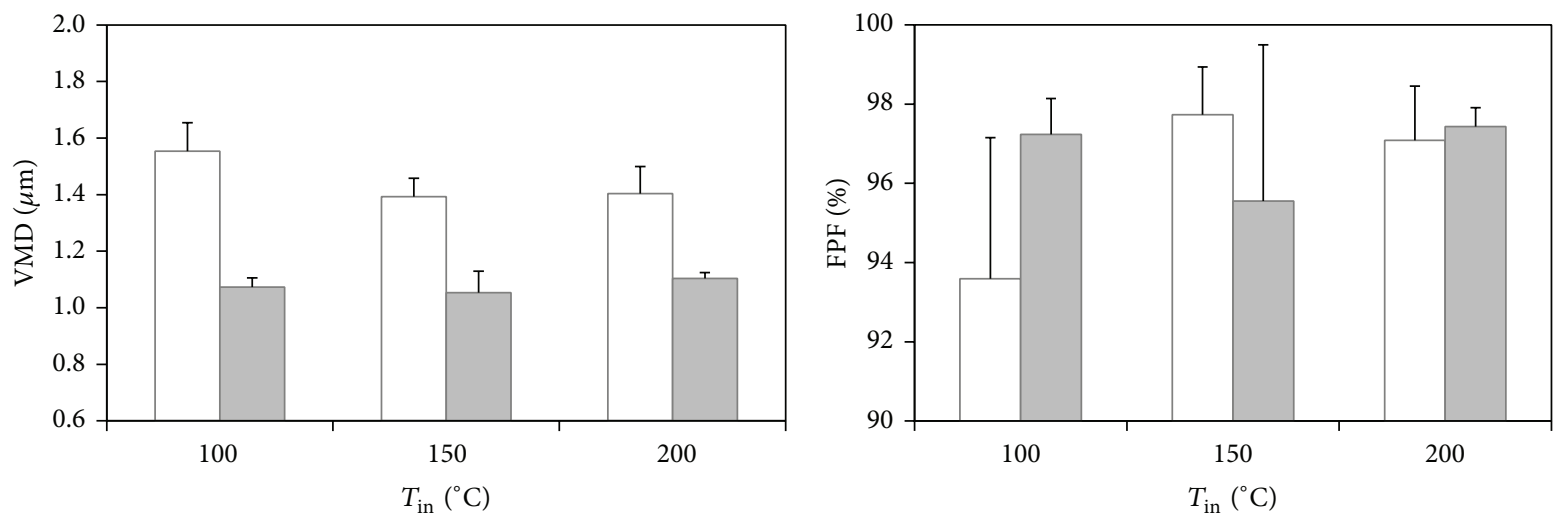

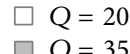

$\square \mathrm{Q}=20$

$\square \mathrm{Q}=35$

FIGURE 7: Volumetric median diameter (VMD) and fraction of fine particles (FPF) for DACMC microparticles as a function of the temperature and the flow rate of the drying air $\left(\mathrm{m}^{3} / \mathrm{hr}\right)$. Error bars denote the standard deviation.

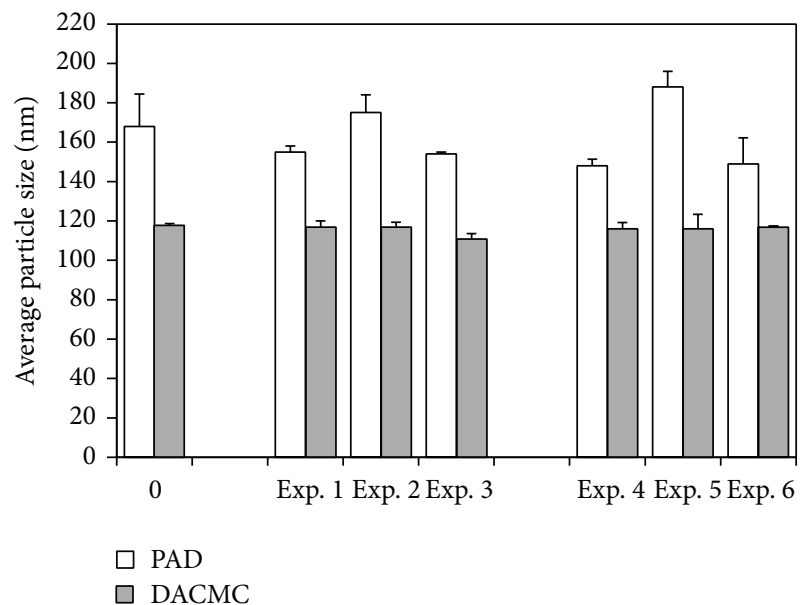

(a)

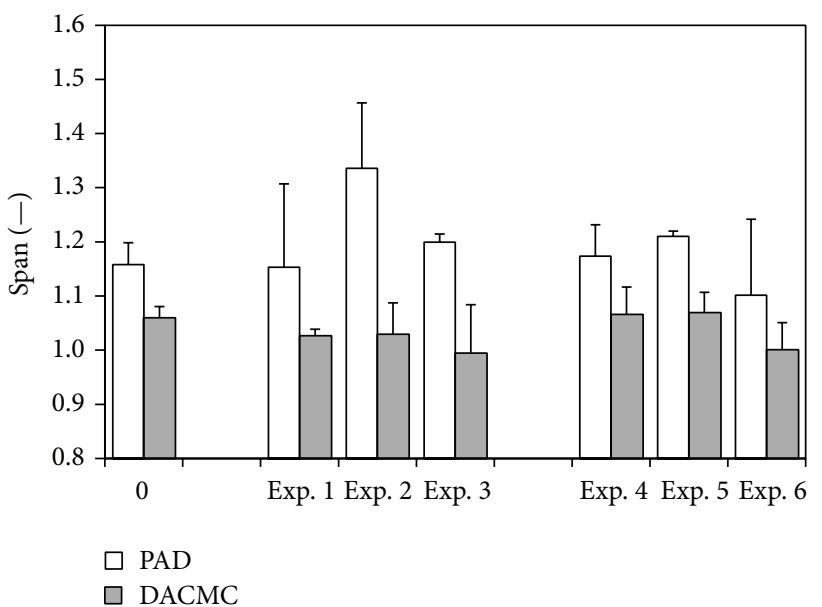

(b)

Figure 8: Average size (a) and span of the distribution (b) for nanoparticles obtained after reconstitution of spray dried powders with water at room temperature. Sample "0" denotes the original nanosuspensions (precursors). Error bars indicate the standard deviations. 
always higher than $80 \%$, suggesting the possibility to deliver almost all the powder emitted from the inhaler into the distal parts of the respiratory system.

The average size of aerosolized PAD particles increases with the drying rate (higher flow and higher temperature of the hot air) which is related to the observed particle deformations. This effect can be explained in two ways as follows.

(1) Since a spherical particle has the lowest volume, so deformation of particle with the given mass always leads to a higher volumetric size.

(2) Particles with corrugated surface can be more difficult to disperse into single particles (due to interlocking effect [29]), so the observed distribution of particle size in the aerosol corresponds not to individual particles but rather to some aggregates which had not been dispersed in the inhaler. Referring to SEM pictures of produced PAD powders (Figure 3 ) it is seen that some small particles $(<1 \mu \mathrm{m})$ are located in the cavities of larger particles. If such structures are not broken apart by the aerodynamic interactions, the relative amount of small particles in the aerosol will be decreased, and it will result in the higher average particle size (VMD).

The situation is different in case of DACMC particles for which the surface deformation caused by drying is weaker. Formation of the external crust can be delayed in this case due to the lower polymer concentration and lower liquid viscosity, so the particle shape almost always remains spherical according to the mechanism shown in Figure 5(b). Some surface defects are observed mainly at the high drying rates. It is found that powders obtained at higher flow rates of the drying air are aerosolized to smaller particles than the ones obtained at the lower airflow rate (Figure 7). The reason for that is not fully clear but the effect may be related to the structural changes of the DACMC nanostructures during the loss of water due to evaporation, which can be dependent on the drying rate.

Finally, the size distribution of nanoparticles reconstituted following the addition of water is compared in Figure 8(a). In general, it is seen that the nanostructure of colloids is restored after hydration of spray dried powders. This observation confirms that material processing during SD does not destroy the initial properties of tested polymers including the capability of self-organization (such as coiling/folding) in the aqueous environment. A scatter of the average particle size observed for PAD nanoparticles in Figure 8(a) cannot be reasonably linked to the drying conditions since the maximum can be found at the moderate inlet air temperatures $\left(T_{\text {in }}=150^{\circ} \mathrm{C}\right)$.

Considering the values of span (2) presented in Figure 8(b), which give more information on the size distribution, it can be concluded that polydispersity of PAD nanoparticles is always higher than the one of DACMC. Particles processed by drying at $150^{\circ} \mathrm{C}$ and rehydration show wider distribution $(S=$ 1.2-1.35 after drying as compared to $S=1.15$ for the precursor). On the other hand, reconstituted nanosuspensions obtained from particles processed at both lower and higher drying temperatures are either less or equally polydisperse as the precursor.

\section{Conclusions}

Presented investigations demonstrate the following.

(i) Nanostructures of biocompatible polymers PAD and DACMC with the average size of $120-170 \mathrm{~nm}$ can be obtained by a relatively simple chemical procedure in aqueous environment.

(ii) Obtained nanoparticles can be conveniently and effectively converted into dry powders using spray drying technique from aqueous suspensions without any additives.

(iii) Selected parameters of SD process, such as inlet temperature and flow rate of drying air (both determining the drying rate), are essential for surface morphology of produced powder particles.

(iv) The resulting powders of both polymers have the adequate properties to be delivered to the lungs via inhalation using a simple and inexpensive capsuletype inhaler available on the market.

(v) After powders reconstitution in water, the initial nanostructures are restored, giving the opportunity to study their suitability as potential drug carriers acting in the sites of deposition of inhaled microparticles.

The proposed methodology forms the general framework for the production of drug-loaded polymeric nanoparticles for pulmonary delivery via inhalative route as micrometric powders. In contrast to the earlier concepts (as the one presented by Tsapis et al. [15]), we use aqueous nanocolloids formed by self-organizing biocompatible polymeric particles which then are dried without any special chemical additives and foreign structures. Further studies focused on the application of the proposed platform for embedding individual drugs intended for treatment of pulmonary diseases using nanosized polymeric carriers are underway.

\section{Conflict of Interests}

The authors declare that there is no conflict of interests regarding the publication of this paper.

\section{Acknowledgments}

The work is financed by statutory activity of the Faculty of Chemical and Process Engineering, Warsaw University of Technology. The input of Katarzyna Jabłczyńska and Tomasz R. Sosnowski is related to their participation in the COST Action MP1106 "Smart and green interfaces: from single bubbles and drops to industrial, environmental and biomedical applications."

\section{References}

[1] E. Fattal, H. Hillaireau, S. Mura, J. Nicolas, and N. Tsapis, “Targeted delivery using biodegradable polymeric nanoparticles," in 
Fundamentals and Applications of Controlled Drug Delivery, J. Siepmann, R. A. Siegel, and M. J. Rathbone, Eds., pp. 255-288, CRS \& Springer, New York, NY, USA, 2012.

[2] J. Panyam and V. Labhasetwar, "Biodegradable nanoparticles for drug and gene delivery to cells and tissue," Advanced Drug Delivery Reviews, vol. 55, no. 3, pp. 329-347, 2003.

[3] F. Danhier, E. Ansorena, J. M. Silva, R. Coco, A. Le Breton, and V. Préat, "PLGA-based nanoparticles: an overview of biomedical applications," Journal of Controlled Release, vol. 161, no. 2, pp. 505-522, 2012.

[4] H. M. Mansour, M. Sohn, A. Al-Ghananeem, and P. P. DeLuca, "Materials for pharmaceutical dosage forms: molecular pharmaceutics and controlled release drug delivery aspects," International Journal of Molecular Sciences, vol. 11, no. 9, pp. 3298-3322, 2010.

[5] P. Sheth and P. B. Myrdal, "Polymers for pulmonary drug delivery," in Controlled Pulmonary Drug Delivery, H. D. C. Smyth and A. J. Hickey, Eds., pp. 265-282, Controlled Release Society \& Springer, New York, NY, USA, 2011.

[6] J. Wei, X.-J. Ju, X.-Y. Zou et al., "Multi-stimuli-responsive microcapsules for adjustable controlled-release," Advanced Functional Materials, vol. 24, no. 22, pp. 3312-3323, 2014.

[7] Y. Lu, W. Sun, and Z. Gu, "Stimuli-responsive nanomaterials for therapeutic protein delivery," Journal of Controlled Release, vol. 194, pp. 1-19, 2014.

[8] J. Fang, H. Nakamura, and H. Maeda, “The EPR effect: unique features of tumor blood vessels for drug delivery, factors involved, and limitations and augmentation of the effect," Advanced Drug Delivery Reviews, vol. 63, no. 3, pp. 136-151, 2011.

[9] S. Acharya and S. K. Sahoo, "PLGA nanoparticles containing various anticancer agents and tumour delivery by EPR effect," Advanced Drug Delivery Reviews, vol. 63, no. 3, pp. 170-183, 2011.

[10] W. Yang, J. I. Peters, and R. O. Williams III, "Inhaled nanoparticles-A current review," International Journal of Pharmaceutics, vol. 356, no. 1-2, pp. 239-247, 2008.

[11] J. C. Sung, B. L. Pulliam, and D. A. Edwards, "Nanoparticles for drug delivery to the lungs," Trends in Biotechnology, vol. 25, no. 12, pp. 563-570, 2007.

[12] S. Newman, Respiratory Drug Delivery: Essential Theory and Practice, RDD Online/VCU, Richmond, VA, USA, 2009.

[13] H. Bisgaard, C. O'Callaghan, and G. C. Smaldone, Eds., Drug Delivery to the Lungs, Marcel Dekker, New York, NY, USA, 2002.

[14] T. R. Sosnowski, A. Moskal, and L. Gradoń, "Dynamics of oropharyngeal aerosol transport and deposition with the realistic flow pattern," Inhalation Toxicology, vol. 18, no. 10, pp. 773780, 2006.

[15] N. Tsapis, D. Bennett, B. Jackson, D. A. Weitz, and D. A. Edwards, "Trojan particles: large porous carriers of nanoparticles for drug delivery," Proceedings of the National Academy of Sciences of the United States of America, vol. 99, no. 19, pp. 1200112005, 2002.

[16] T. R. Sosnowski, "Nanosized and nanostructured particles in pulmonary drug delivery," Journal of Nanoscience and Nanotechnology, vol. 15, no. 5, pp. 3476-3487, 2015.

[17] H. M. Mansour, Y.-S. Rhee, and X. Wu, "Nanomedicine in pulmonary delivery," International Journal of Nanomedicine, vol. 4, pp. 299-319, 2009.

[18] L. Gradoń and T. R. Sosnowski, "Formation of particles for dry powder inhalers," Advanced Powder Technology, vol. 25, no. 1, pp. $43-55,2014$.
[19] R. Vehring, "Expert review: pharmaceutical particle engineering via spray drying," Pharmaceutical Research, vol. 25, no. 5, pp. 999-1022, 2008.

[20] K. Kramek-Romanowska, M. Odziomek, T. R. Sosnowski, and L. Gradoń, "Effects of process variables on the properties of spray-dried mannitol and mannitol/disodium cromoglycate powders suitable for drug delivery by inhalation," Industrial and Engineering Chemistry Research, vol. 50, no. 24, pp. 13922-13931, 2011.

[21] A. B. D. Nandiyanto and K. Okuyama, "Progress in developing spray-drying methods for the production of controlled morphology particles: from the nanometer to submicrometer size ranges," Advanced Powder Technology, vol. 22, no. 1, pp. 1-19, 2011.

[22] M. Odziomek, T. R. Sosnowski, and L. Gradoń, "Conception, preparation and properties of functional carrier particles for pulmonary drug delivery," International Journal of Pharmaceutics, vol. 433, no. 1-2, pp. 51-59, 2012.

[23] J. Maia, M. B. Evangelista, H. Gil, and L. Ferreira, "Dextranbased materials for biomedical applications," in Carbohydrates Applications in Medicine, M. H. Gil, Ed., pp. 31-53, Research Signpost, Kerala, India, 2014.

[24] L. Jiang, Y. Li, L. Zhang, and X. Wang, "Preparation and characterization of a novel composite containing carboxymethyl cellulose used for bone repair," Materials Science and Engineering C, vol. 29, no. 1, pp. 193-198, 2009.

[25] C. Mu, J. Guo, X. Li, W. Lin, and D. Li, "Preparation and properties of dialdehyde carboxymethyl cellulose crosslinked gelatin edible films," Food Hydrocolloids, vol. 27, no. 1, pp. 2229, 2012.

[26] H. Zhao and N. D. Heindel, "Determination of degree of substitution of formyl groups in polyaldehyde dextran by the hydroxylamine hydrochloride method," Pharmaceutical Research, vol. 8, no. 3, pp. 400-402, 1991.

[27] USP General Chapters, USP ;601> Aerosols, Nasal Sprays, Metered-Dose Inhalers, and Dry Powder Inhalers, USP 32-NF27, US Pharmacopeial Convention, Rockville, Md, USA, 2009.

[28] J. M. Gac and L. Gradoń, "A distributed parameter model for the spray drying of multicomponent droplets with a crust formation," Advanced Powder Technology, vol. 24, no. 1, pp. 324330, 2013.

[29] X. M. Zeng, G. P. Martin, and C. Marriott, Particulate Interactions in Dry Powder Formulations for Inhalation, CRC Press, London, UK, 2000. 

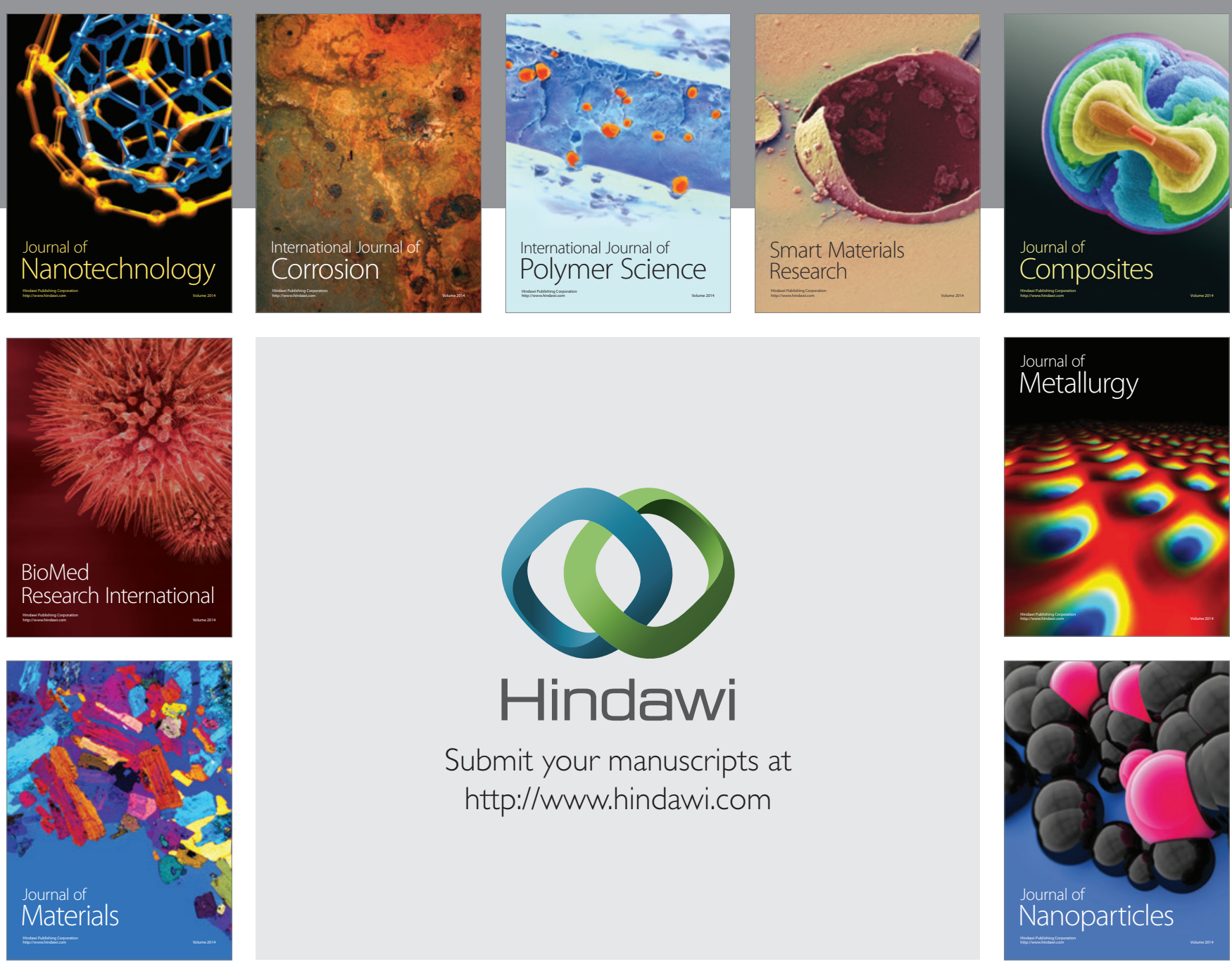

Submit your manuscripts at http://www.hindawi.com
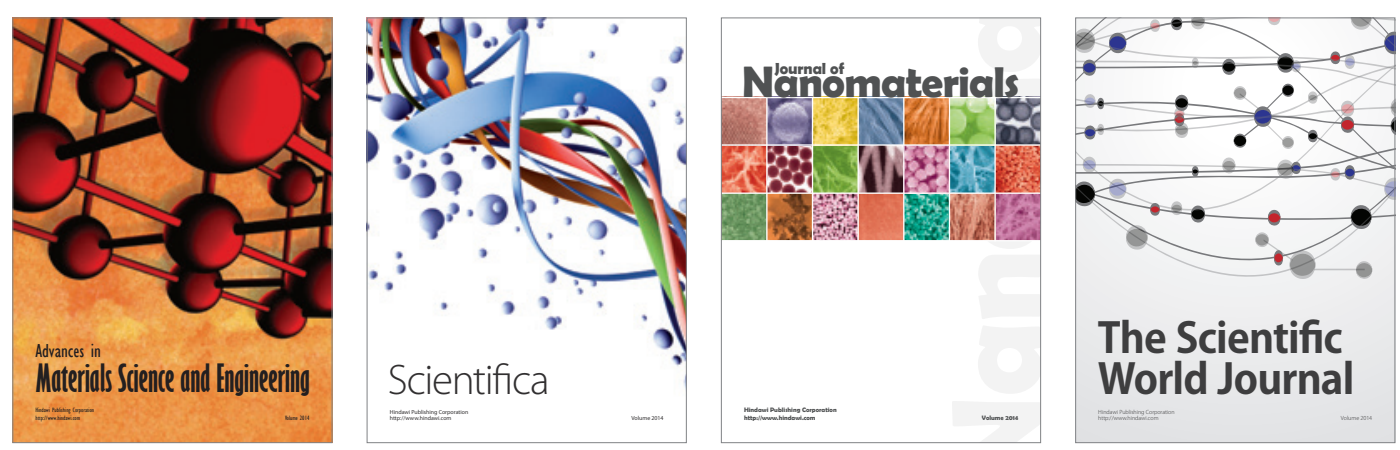

\section{The Scientific World Journal}
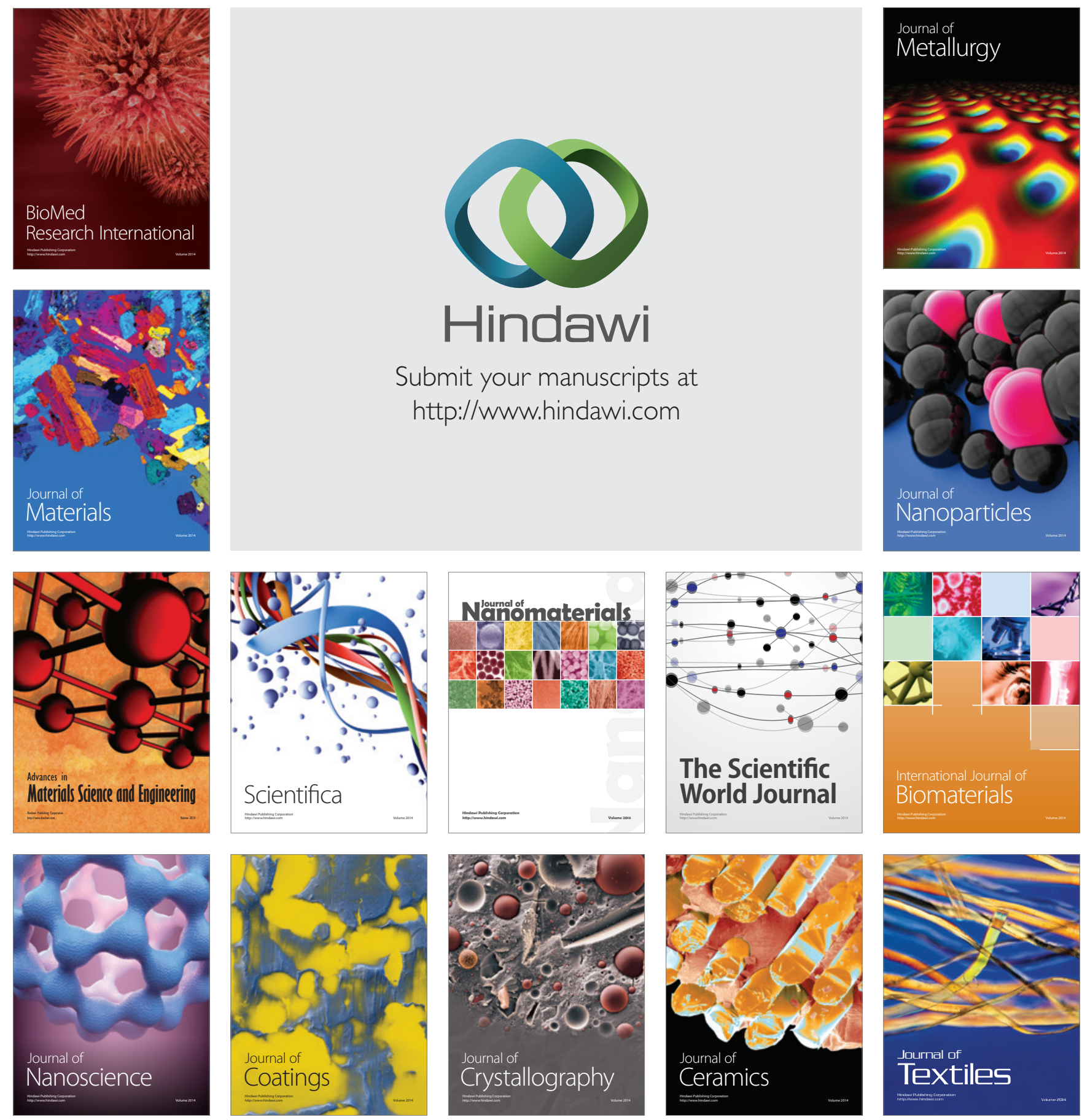